\title{
From Heisenberg to Gödel via Chaitin ${ }^{1}$
}

\author{
Cristian S. Calude ${ }^{2}$ and Michael A. Stay ${ }^{2}$
}

Received February 1, 2004; accepted April 1, 2004

Published Online: August 23, 2007

\begin{abstract}
In 1927 Heisenberg discovered that the "more precisely the position is determined, the less precisely the momentum is known in this instant, and vice versa." Four years later Gödel showed that a finitely specified, consistent formal system which is large enough to include arithmetic is incomplete. As both results express some kind of impossibility it is natural to ask whether there is any relation between them, and, indeed, this question has been repeatedly asked for a long time. The main interest seems to have been in possible implications of incompleteness to physics. In this note we will take interest in the converse implication and will offer a positive answer to the question: Does uncertainty imply incompleteness? We will show that algorithmic randomness is equivalent to a "formal uncertainty principle" which implies Chaitin's informationtheoretic incompleteness. We also show that the derived uncertainty relation, for many computers, is physical. In fact, the formal uncertainty principle applies to all systems governed by the wave equation, not just quantum waves. This fact supports the conjecture that uncertainty implies algorithmic randomness not only in mathematics, but also in physics.
\end{abstract}

KEY WORDS: algorithmic randomness; uncertainty; incompleteness.

In mathematics you don't understand things.

You just get used to them.- - J. von Neumann

\section{INTRODUCTION}

Are there any connections between uncertainty and incompleteness? We don't know of any reaction of Heisenberg to this question. However, Gödel's hostility to any suggestion regarding possible connections between his incompleteness theorem and physics, particularly, Heisenberg's uncertainty relation, is

\footnotetext{
${ }^{1}$ Partially supported by the Vice-Chancellor's University Development Fund 23124.

${ }^{2}$ Department of Computer Science, The University of Auckland, New Zealand; e-mail: \{cristian,msta039\}@ec.auckland.ac.nz.

Originally published in International Journal of Theoretical Physics, Vol. 44, No. 7, 2005, Due to a publishing error, authorship of the article was credited incorrectly. The corrected article is reprinted in its entirety here. The online version of the original article can be found at http://dx.doi.org/10.1007/s10773-005-7081-8
} 
well-known. ${ }^{3}$ One of the obstacles in establishing such a connection comes from the different nature of these two results: uncertainty is a quantitative phenomenon while incompleteness is prevalently qualitative.

In recent years there have been a lot of interest in the relations between computability and incompleteness and physics. Opinions vary considerably, from the conclusion that the impact on Gödel and Turing incompleteness theorems to physics is a red herring (see Casti and Traub, 1994; Casti and Karlquist, 1996), to Hawking's view that "a physical theory is self-referencing, like in Gödel's theorem. ... Theories we have so far are both inconsistent and incomplete" (cf. Hawking, 2002). A very interesting analysis of the possible impact of Gödel's incompleteness theorems in physics was written by Barrow (1998, 2000); the prevalence of physics over mathematics is argued by Deutsch (1997); for Svozil (2003, 2004), Heisenberg's incompleteness is pre-Gödelian-Turing and finite. Other relevant papers are Geroch and Hartle (1986), Peres (1985), and Peres and Zurek (1982).

In this note we do not ask whether Gödel's incompleteness has any bearing on Heisenberg's uncertainty, but the converse: Does uncertainty imply incompleteness? We will show that we can get a positive answer to this question: algorithmic randomness can be recast as a "formal uncertainty principle" which implies Chaitin's information-theoretic version of Gödel's incompleteness.

\section{OUTLINE}

We begin with overviews of the relevant ideas first discovered by Heisenberg, Gödel, and Chaitin.

Next, we show that random reals, of which Chaitin Omega numbers are just an example, satisfy a "formal uncertainty principle," namely

$$
\Delta_{s} \cdot \Delta_{C}\left(\omega_{1} \ldots \omega_{s}\right) \geq \varepsilon
$$

where $\varepsilon$ is a fixed positive constant.

The two conjugate coordinates are the random real and the binary numbers describing the programs that generate its prefixes. Then, the uncertainty in the random real given an $n$-bit prefix is $2^{-n}$, and the uncertainty in the size of the shortest program that generates it is, to within a multiplicative constant, $2^{n}$.

The Fourier transform is a lossless transformation, so all the information contained in the delta function $\delta_{\Omega(x)}=1$ if $x=\Omega, \delta_{\Omega(x)}=0$, otherwise, is preserved in the conjugate. Therefore, if you need $n$ bits of information to describe a square wave convergent on the delta function, there must be $n$ bits of information in the Fourier transform of the square wave. Since both the information in the transformed square wave and the shortest program describing the square wave increase linearly with $n$, there is an equivalence between the two.

\footnotetext{
${ }^{3} \mathrm{~J}$. Wheeler was thrown out of Gödel's office for asking the question "Professor Gödel, what connection do you see between your incompleteness theorem and Heisenberg's uncertainty principle?", cf. Chaitin's account cited in Barrow ((1998), p. 221.)
} 
We show that the formal uncertainty principle is a true uncertainty principlethat is, the terms are bounded by the standard deviations of two random variables with particular probability distributions. We note that for many self-delimiting Turing machines $C$, the halting probability $\Omega_{C}$ is computable; in these cases, there are quantum systems with observables described by these probability distributions, and our uncertainty relation is equivalent to Heisenberg's.

Finally, (1) implies a strong version of Gödel's incompleteness, Chaitin's information-theoretic version (Chaitin, 1975a,b) (see also the analysis in Delahaye (1994); Calude (2002a)). Chaitin's proof relied on measure theory; we present here a new proof via a complexity-theoretic argument.

\section{HEISENBERG}

In 1925 Heisenberg developed the theory of matrix mechanics; it was his opinion that only observable quantities should play any role in a theory. At the time, all observations came in the form of spectral absorption and emission lines. Heisenberg, therefore, considered the "transition quantities" governing the jumps between energy states to be the fundamental concepts of his theory. Together with Born, who realized Heisenberg's transition rules obeyed the rules of matrix calculus, he developed his ideas into a theory that predicted nearly all the experimental evidence available.

The next year, Schrödinger introduced what became known as wave mechanics, together with a proof that the two theories were equivalent. Schrödinger argued that his version of quantum mechanics was better in that one could visualize the behavior of the electrons in the atom. Many other physicists agreed with him.

Schrödinger's approach disgusted Heisenberg; in a letter to Pauli (see Pauli, 1979), he called Schrödinger's interpretation “crap.” Publicly, however, he was more restrained. In Heisenberg (1926) he argued that while matrix mechanics was hard to visualize, Schrödinger's interpretation of wave mechanics was selfcontradictory, and concluded that something was still missing from the interpretation of quantum theory.

In 1927 Heisenberg published "Über den Anschaulichen Inhalt der Quantentheoretischen Kinematik und Mechanik" (see Heisenberg, 1927) to provide the missing piece. First, he gave his own definition of visualization: "We believe we have gained intuitive understanding of a physical theory, if in all simple cases, we can grasp the experimental consequences qualitatively and see that the theory does not lead to any contradictions." In this sense, matrix mechanics was just as intuitive as wave mechanics. Next, he argued that terms like "the position of a particle" can only make sense in terms of the experiment that measures them.

To illustrate, he considered the measurement of an electron by a microscope. ${ }^{4}$ The accuracy is limited by the wavelength of the light illuminating the electron;

\footnotetext{
${ }^{4}$ Heisenberg might have been so concerned with uncertainty because in 1923 he almost failed his Ph.D. exam when Sommerfeld asked about (optical) limitations to the resolution of the microscope.
} 
one can use as short a wavelength as one wishes, but for very short wavelengths, the Compton effect is non-negligible. He wrote, (see Heisenberg, 1927, pp. 174-175),

At the instant of time when the position is determined, that is, at the instant when the photon is scattered by the electron, the electron undergoes a discontinuous change in momentum. This change is the greater the smaller the wavelength of the light employed, i.e., the more exact the determination of the position. At the instant at which the position of the electron is known, its momentum therefore can be known only up to magnitudes which correspond to that discontinuous change; thus, the more precisely the position is determined, the less precisely the momentum is known, and conversely.

Heisenberg estimated the uncertainty to be on the order

$$
\delta_{p} \cdot \delta_{q} \sim \hbar,
$$

where $\hbar$ is Planck's constant over $2 \pi$.

Kennard (see Kennard, 1927) was the first to publish the uncertainty relation in its exact form. He proved in 1927 that for all normalized state vectors $|\Psi\rangle$,

$$
\Delta_{p} \cdot \Delta_{q} \geq \hbar / 2,
$$

where $\Delta_{p}$ and $\Delta_{q}$ are standard deviations of momentum and position, i.e.

$$
\Delta_{p}^{2}=\left\langle\Psi\left|p^{2}\right| \Psi\right\rangle-\langle\Psi|p| \Psi\rangle^{2} ; \Delta_{q}^{2}=\left\langle\Psi\left|q^{2}\right| \Psi\right\rangle-\langle\Psi|q| \Psi\rangle^{2} .
$$

Thus, assuming quantum mechanics is an accurate description of reality, the formalism is compatible with Heisenberg's principle.

\section{GÖDEL}

In 1931 Gödel published his (first) incompleteness theorem in Gödel (1931) (see also Feferman et al., 1986, 1990). According to the current terminology, he showed that every formal system which is (1) finitely specified, (2) rich enough to include the arithmetic, and (3) consistent, is incomplete. That is, there exists an arithmetical statement which (A) can be expressed in the formal system, (B) is true, but $(\mathrm{C})$ is unprovable within the formal system.

All conditions are necessary. Condition (1) says that there is an algorithm listing all axioms and inference rules (which could be infinite). Taking as axioms all true arithmetical statements will not do, as this set is not finitely listable. A "true arithmetical statement" is a statement about non-negative integers which cannot be invalidated by finding any combination of non-negative integers that contradicts it. Condition (2) says that the formal systems has all the symbols and axioms used in arithmetic, the symbols for 0 (zero), $S$ (successor), + (plus), $\times$ (times), $=$ (equality) and the axioms making them work (as for example, $x+S(y)=$ $S(x+y)$ ). Condition (2) cannot be satisfied if you do not have individual terms for $0,1,2, \ldots$; for example, Tarski (1994) proved that the plane Euclidean geometry, 
which refers to points, circles and lines, is complete. ${ }^{5}$ Finally (3) means that the formal system is free of contradictions.

Like uncertainty, incompleteness has provoked a lot of interest (and abuse).

\section{CHAITIN}

Chaitin has obtained three types of information-theoretic incompleteness results (scattered through different publications, Chaitin, 1975a,b, 1982, 1992; see also Chaitin, 1999, 2002). The strongest form concerns the computation of the bits of a Chaitin Omega number $\Omega_{U}$, the halting probability of a self-delimiting universal Turing machine $U$ (see also the analysis in Delahaye, 1994; Calude, 2002a). A self-delimiting Turing machine $C$ is a normal Turing machine $C$ which processes binary strings into binary strings and has a prefix-free domain, that is, if $C(x)$ is defined and $y$ is either a proper prefix or an extension of $x$, then $C(y)$ is not defined. The self-delimiting Turing machine $U$ is universal if for every selfdelimiting Turing machine $C$ there exists a fixed binary string $p$ (the simulator) such that for every input $x, U(p x)=C(x)$ : either both computations $U(p x)$ and $C(x)$ stop and, in this case they produce the same output or both computations never stop. The Omega number introduced in Chaitin (1975a)

$$
\Omega_{U}=0 . \omega_{1} \omega_{2} \ldots \omega_{n} \ldots
$$

is the halting probability of $U$; it is one of the most important concepts in algorithmic information theory (see Calude, 2002a).

Chaitin (1975a) proved the following result: Assume that $X$ is a formal system satisfying conditions (1), (2) and (3) in Gödel's incompleteness theorem. Then,for every self-delimiting universal Turing machine $U, X$ can determine the positions and values of only finitely scattered bits of $\Omega_{U}$, and one can give a bound on the number of bits of $\Omega_{U}$ which $X$ can determine. This is a form of incompleteness because, with the exception of finitely many $n$, any true statement of the form "the $n$th bit of $\Omega_{U}$ is $\omega_{n}$ " is unprovable in $X$.

For example, we can take $X$ to be $Z F C^{6}$ under the assumption that it arithmetically sound, that is, any theorem of arithmetic proved by $Z F C$ is true. Solovay (2000) has constructed a specific self-delimiting universal Turing machine $S$ (called Solovay machine) such that ZFC cannot determine any bit of $\Omega_{S}$. In this way one can obtain constructive versions of Chaitin's theorem. For example, if $Z F C$ is arithmetically sound and $S$ is a Solovay machine, then the statement "the 0 th bit of the binary expansion of $\Omega_{S}$ is 0 " is true but unprovable in ZFC. In fact,

\footnotetext{
${ }^{5}$ This result combined with with Gödel's completeness theorem implies decidability: there is an algorithm which accepts as input an arbitrary statement of plane Euclidean geometry, and outputs "true" if the statement is true, and "false" if it is false. The contrast between the completeness of plane Euclidean geometry and the incompleteness of arithmetic is striking.

${ }^{6}$ Zermelo-Fraenkel set theory with choice.
} 
one can effectively construct arbitrarily many examples of true and unprovable statements of the above form, cf. Calude (2002b).

\section{RUDIMENTS OF ALGORITHMIC INFORMATION THEORY}

In this section we will present some basic facts of algorithmic information theory in a slightly different form which is suitable for the results appearing in the following section.

We will work with binary strings; the length of the string $x$ is denoted by $|x|$. For every $n \geq 0$ we denote by $B(n)$ the binary representation of the number $n+1$ without the leading 1 . For example, $0 \mapsto \lambda$ (the empty string), $1 \mapsto 0,2 \mapsto 1,3 \mapsto 00, \ldots$ The length of $B(n)$ is almost equal to $\log _{2}(n)$; more precisely, it is $\left\lfloor\log _{2}(n+1)\right\rfloor$. The function $B$ is bijective and we denote by $N$ its inverse. The string $x$ is length-lexicographically less than the string $y$ if and only if $N(x)<N(y)$.

We need first the Kraft-Chaitin theorem: Let $n_{1}, n_{2}, \ldots$ be a computable sequence of non-negative integers such that

$$
\sum_{i=1}^{\infty} 2^{-n_{i}} \leq 1 .
$$

Then, we can effectively construct a prefix-free sequence of strings (that is, no $w_{i}$ is a proper prefix of any $w_{j}$ with $\left.i \neq j\right) w_{1}, w_{2}, \ldots$ such that for each $i \geq$ $1,\left|w_{i}\right|=n_{i}$.

Let $C$ be a self-delimiting Turing machine. The program-size complexity induced by $C$ is defined by $H_{C}(x)=\min \{|w| \mid C(w)=x\}$ (with the convention that strings not produced by $C$ have infinite complexity). One might suppose that the complexity of a string would vary greatly between choices of self-delimiting Turing machine. However, because of the universality requirement, the complexity difference between $C$ and $C^{\prime}$ is at most the length of the shortest program for $C^{\prime}$ that simulates $C$. Therefore, the complexity of a string is fixed to within an additive constant. This is known as the "invariance theorem" (see Calude, 2002a), and is usually stated: For every self-delimiting universal Turing machine $U$ and selfdelimiting Turing machine $C$ there exists a constant $\varepsilon>0$ (which depends upon $U$ and $C$ ) such that for every string $x$,

$$
H_{U}(x) \leq \varepsilon+H_{C}(x) .
$$

For our aim it is more convenient to define the complexity measure $\nabla_{C}(x)=\min \{N(w) \mid C(w)=x\}$, the smallest integer whose binary representation produces $x$ via $C$. Clearly, for every string $x$,

$$
2^{H_{C}(x)}-1 \leq \nabla_{C}(x)<2^{H_{C}(x)+1}-1 .
$$


Therefore we can say that $\Delta_{C}(x)$, our uncertainty in the value $\nabla_{C}(x)$, is the difference between the upper and lower bounds given, namely $\Delta_{C}(x)=2^{H_{C}(x)}$.

The invariance theorem can now be stated as follows: for every self-delimiting universal Turing machine $U$ and self-delimiting Turing machine $C$ there exists a constant $\varepsilon>0$ (which depends upon $U$ and $C$ ) such that for every string $x$,

$$
\Delta_{U}(x) \leq \varepsilon \cdot \Delta_{C}(x) .
$$

Let $\Delta_{s}=2^{-s}$. Chaitin's theorem (see Chaitin, 1975a) stating that the bits of $\Omega_{U}$ in (2) form a random sequence can now be presented as a "formal uncertainty principle": for every self-delimiting Turing machine $C$ there is a constant $\varepsilon>0$ (which depends upon $U$ and $C$ ) such that

$$
\Delta_{s} \cdot \Delta_{C}\left(\omega_{1} \ldots \omega_{s}\right) \geq \varepsilon
$$

The inequality (4) is an uncertainty relation as it reflects a limit to which we can simultaneously increase both the accuracy with which we can approximate $\Omega_{U}$ and the complexity of the initial sequence of bits we compute; it relates the uncertainty of the output to the size of the input. When $s$ grows indefinitely, $\Delta_{S}$ tends to zero, in contrast with $\Delta_{C}\left(\omega_{1} \ldots \omega_{s}\right)$ which tends to infinity; their product is not only bounded from below, but increases indefinitely (see also (6)). From a complexity viewpoint (4) tells us that there is a limit $\varepsilon$ up to which we can uniformly compress the initial prefixes of the binary expansion of $\Omega_{U}$.

How large can be $\varepsilon$ in (4)? For example, $\varepsilon=1$ when $C=U_{0}$ is a special universal self-delimiting Turing machine:

$$
\Delta_{s} \cdot \Delta_{U_{0}}\left(\omega_{1} \ldots \omega_{s}\right) \geq 1
$$

If $U$ is universal and satisfies (4), then a universal machine $U_{0}$ satisfying (5) can be defined by $U_{0}\left(0^{\varepsilon} x\right)=U(x)$ (so requiring that any input to $U_{0}$ not starting with $\varepsilon$ zeros causes the machine to go into an infinite loop).

In fact, in view of the strong complexity-theoretic characterization of random sequences (see Chaitin, 1975a; Calude, 2002a) a stronger form of (4) is true: for every positive integer $N$ there is a bound $M$ (which depends upon $U, C$ and $N$ ) such that for all $s \geq M$ we have:

$$
\Delta_{s} \cdot \Delta_{C}\left(\omega_{1} \ldots \omega_{s}\right) \geq N
$$

The constant $N$ appearing in (4) can be made arbitrarily large in case $s$ is large enough; the price paid appears in the possible violation of the inequality for the first $s<M$ bits.

Is (4) a 'true' uncertainty relation? We prove that the variables $\Delta_{s}$ and $\Delta_{C}$ in (4) are standard deviations of two measurable observables in suitable probability spaces. 
For $\Delta_{s}$ we consider the space of all real numbers in the unit interval which are approximated to exactly $s$ digits. Consider the probability distribution $\operatorname{Prob}(v)=$ $P_{C}(v) / \Omega_{C}^{s}$, where $P_{C}(x)=\sum_{C(y)=x} 2^{-|y|}$ and $\Omega_{C}^{s}=\sum_{|x|=s} P_{C}(x)$.

Now fix the first $s$ digits of $\Omega_{U}, \omega_{1} \omega_{2} \ldots \omega_{s}$ and define

$$
\alpha=2^{-s / 2} \cdot\left(\operatorname{Prob}\left(\omega_{1} \omega_{2} \ldots \omega_{s}\right)\right)^{-1 / 2} \cdot\left(1-\operatorname{Prob}\left(\omega_{1} \omega_{2} \ldots \omega_{s}\right)\right)^{-1 / 2} .
$$

The random variable $X$ on a real approximated by the first $s$ digits $v=v_{1} v_{2} \ldots v_{s}$ is defined by the delta function $X(v)=\alpha$ if $v=\omega_{1} \omega_{2} \ldots \omega_{s}$ and $X(v)=0$ otherwise. Then the expectation values of $X$ and $X^{2}$ are $\langle X\rangle=\alpha \cdot \operatorname{Prob}\left(\omega_{1} \omega_{2} \ldots \omega_{s}\right)$ and $\left\langle X^{2}\right\rangle=\alpha^{2} \cdot \operatorname{Prob}\left(\omega_{1} \omega_{2} \ldots \omega_{s}\right)$, so the standard deviation is $\sigma_{X}=\Delta_{s}$.

For $\Delta_{C}$ we consider

$\beta=\left(\Delta_{C}\left(\omega_{1} \omega_{2} \ldots \omega_{s}\right)\right)^{1 / 2} \cdot\left(\operatorname{Prob}\left(\omega_{1} \omega_{2} \ldots \omega_{s}\right)\right)^{-1 / 2} \cdot\left(1-\operatorname{Prob}\left(\omega_{1} \omega_{2} \ldots \omega_{s}\right)\right)^{-1 / 2}$,

and the same space but the random variable $Y\left(\omega_{1} \omega_{2} \ldots \omega_{s}\right)=\beta$ and $Y(v)=$ 0 if $v \neq \omega_{1} \omega_{2} \ldots \omega_{s}$. Then, the expectation values of $Y$ and $Y^{2}$ are $\langle Y\rangle=\beta$. $\operatorname{Prob}\left(\omega_{1} \omega_{2} \ldots \omega_{s}\right)$ and $\left\langle Y^{2}\right\rangle=\beta^{2} \cdot \operatorname{Prob}\left(\omega_{1} \omega_{2} \ldots \omega_{s}\right)$, so the standard deviation is $\sigma_{Y}=\Delta_{C}\left(\omega_{1} \omega_{2} \ldots \omega_{s}\right)$.

Hence the relation (4) becomes:

$$
\sigma_{X} \cdot \sigma_{Y}=\Delta_{s} \cdot \Delta_{C}\left(\omega_{1} \omega_{2} \ldots \omega_{s}\right) \geq \varepsilon,
$$

so for $U_{0}$ satisfying (5) we have:

$$
\sigma_{X} \cdot \sigma_{Y} \geq 1
$$

\section{FROM HEISENBERG TO CHAITIN}

Since self-delimiting universal Turing machines are strictly more powerful than non-universal ones, the inequality holds for the weaker computers as well. In many of these cases, the halting probability of the machine is computable, and we can construct a quantum algorithm to produce a set of qubits whose state is described by the distribution.

To illustrate, we consider a quantum algorithm with two parameters, $C$ and $s$, where $C$ is a Turing machine for which the probability of producing each $s$-bit string is computable. We run the algorithm to compute that distribution on a quantum computer with $s$ ouput qubits; it puts the output register into a superposition of spin states, where the probability of each state $|v\rangle$ is $P_{C}(v) / \Omega_{C}^{s}$. Next, we apply the Hamiltonian operator $H=\beta\left|\omega_{1} \ldots \omega_{s}\right\rangle\left\langle\omega_{1} \ldots \omega_{s}\right|$ to the prepared state. A measurement of energy will give $\beta$ with probability $P=\operatorname{Prob}\left(\omega_{1} \omega_{2} \ldots \omega_{s}\right)$ and zero with probability $1-P$. The expectation value for energy, therefore, is exactly the same as that of $Y$, but with units of energy, i.e.

$$
\Delta_{C}\left(\omega_{1} \omega_{2} \ldots \omega_{s}\right)[J] \cdot \Delta_{s} \geq \varepsilon[J],
$$


where $[J]$ indicates Joules of energy.

Now define

$$
\Delta_{t} \equiv \frac{\sigma_{Q}}{|d\langle Q\rangle / d t|},
$$

where $Q$ is any observable that does not commute with the Hamiltonian; that is, $\Delta_{t}$ is the time it takes for the expectation value of $Q$ to change by one standard deviation. With this definition, the following is a form of Heisenberg's uncertainty principle:

$$
\Delta_{E} \cdot \Delta_{t} \geq \hbar / 2
$$

We can replace $\Delta_{E}$ by $\Delta_{C}\left(\omega_{1} \omega_{2} \ldots \omega_{s}\right)$ by the analysis above; but what about $\Delta_{t}$ ? If we choose a time scale such that our two uncertainty relations are equivalent for a single quantum system corresponding to a computer $C$ and one value of $s$, then the relation holds for $C$ and any value of $s$ :

$$
\Delta_{C}\left(\omega_{1} \omega_{2} \ldots \omega_{s}\right)[J] \cdot \Delta_{s} \frac{\hbar}{2 \varepsilon}\left[J^{-1} \cdot J s\right] \geq \frac{\hbar}{2}[J s] .
$$

In this sense, we claim that Heisenberg's uncertainty relation is equivalent to (4). We cannot say whether (4) is physical for universal self-delimiting Turing machines; to do so requires deciding the Church-Turing thesis for quantum systems.

The uncertainty principle now says that getting one more bit of $\Omega_{U}$ requires (asymptotically) twice as much energy. Note, however, that we have made an arbitrary choice to identify energy with complexity. We could have chosen to create a system in which the position of a particle corresponded to the complexity, while momentum corresponded to the accuracy of $C$ 's estimate of $\Omega_{U}$. In that case, the uncertainty in the position would double for each extra bit. Any observable can play either role, with a suitable choice of units.

If this were the only physical connection, one could argue that the result is merely an analogy and nothing more. However, consider the following: let $\rho$ be the density matrix of a quantum state. Let $R$ be a computable positive operator-valued measure, defined on a finite dimensional quantum system, whose elements are each labeled by a finite binary string. Then the statistics of outcomes in the quantum measurement is described by $R: R\left(\omega_{1} \ldots \omega_{s}\right)$ is the measurement outcome and $\operatorname{tr}\left(\rho R\left(\omega_{1} \ldots \omega_{s}\right)\right)$ is the probability of getting that outcome when we measure $\rho$. Under these hypotheses, Tadaki's inequality (1) (see Tadaki, 2002, p. 2), and our inequality (4) imply the existence of a constant $\tau$ (depending upon $R$ ) such that for all $\rho$ and $s$ we have:

$$
\Delta_{s} \cdot \frac{1}{\operatorname{tr}\left(\rho R\left(\omega_{1} \ldots \omega_{s}\right)\right)} \geq \tau .
$$

In other words, there is no algorithm that, for all $s$, can produce 
1. an experimental setup to produce a quantum state and

2. a POVM with which to measure the state such that

3. the probability of getting the result $\omega_{1} \omega_{2} \ldots \omega_{s}$ is greater than $1 /\left(\tau 2^{s}\right)$.

Finally, it is interesting to note that a Fourier transform of the wave function switches between an "Omega space" and a "complexity space." We plan on examining this relationship further in a future paper.

\section{FROM CHAITIN TO GÖDEL}

In this section we prove that the uncertainty relation (4) implies incompleteness.

We start with the following theorem: Fix a universal self-delimiting Turing machine $U$. Let $x_{1} x_{2}$... be a binary infinite sequence and let $F$ be a strictly increasing function mapping positive integers into positive integers. If the set $\left\{\left(F(i), x_{F(i)}\right) \mid i \geq 1\right\}$ is computable, then there exists a constant $\varepsilon>0$ (which depends upon $U$ and the characteristic function of the above set) such that for all $k \geq 1$ we have:

$$
\Delta_{U}\left(x_{1} x_{2} \ldots x_{F(k)}\right) \leq \varepsilon \cdot 2^{F(k)-k} .
$$

To prove (7) we consider for every $k \geq 1$ the strings

$$
w_{1} x_{F(1)} w_{2} x_{F(2)} \ldots w_{k} x_{F(k)},
$$

where each $w_{j}$ is a string of length $F(j)-F(j-1)-1, F(0)=0$, that is, all binary strings of length $F(k)$ where we have fixed bits at the positions $F(1), \ldots, F(k)$.

It is clear that $\sum_{i=1}^{k}\left|w_{i}\right|=F(k)-k$ and the mapping $\left(w_{1}, w_{2}, \ldots, w_{k}\right) \mapsto$ $w_{1} w_{2} \ldots w_{k}$ is bijective, hence to generate all strings of the form (8) we only need to generate all strings of length $F(k)-k$.

Next we consider the enumeration of all strings of the form (8) for $k=$ $1,2, \ldots$ The lengths of these strings will form the sequence

$$
\underbrace{F(1), F(1), \ldots, F(1)}_{2^{F(1)-1} \text { times }}, \ldots \underbrace{, F(k), F(k), \ldots, F(k)}_{2^{F(k)-k} \text { times }}, \ldots
$$

which is computable and satisfies the inequality (3) as

$$
\sum_{k=1}^{\infty} 2^{F(k)-k} \cdot 2^{-F(k)}=1 .
$$

Hence, by Kraft-Chaitin theorem, for every string $w$ of length $F(k)-k$ there effectively exists a string $z_{w}$ having the same length as $w$ such that the set $\left\{z_{w}|| w \mid=F(k)-k, k \geq 1\right\}$ is prefix-free. Indeed, from a string $w$ of length 
$F(k)-k$ we get a unique decomposition $w=w_{1} \ldots w_{k}$, and $z_{w}$ as above, so we can define $C\left(z_{w}\right)=w_{1} x_{F(1)} w_{2} x_{F(2)} \ldots w_{k} x_{F(k)} ; C$ is a self-delimiting Turing machine. Clearly,

$$
\begin{aligned}
\Delta_{C}\left(w_{1} x_{F(1)} w_{2} x_{F(2)} \ldots w_{k} x_{F(k)}\right) & \leq \nabla_{C}\left(w_{1} x_{F(1)} w_{2} x_{F(2)} \ldots w_{k} x_{F(k)}\right) \\
& \leq N\left(z_{w}\right) \leq 2^{F(k)-k+1}-1
\end{aligned}
$$

for all $k \geq 1$. In particular, $\Delta_{C}\left(x_{1} \ldots x_{F(k)}\right) \leq 2^{F(k)-k+1}-1$, so by the invariance theorem we get the inequality (7).

It is easy to see that under the hypothesis of the above theorem the uncertainty relation (4) is violated, so the sequence $x_{1} x_{2} \ldots x_{n} \ldots$ is not random. Indeed, if the sequence were random, then the formal uncertainty principle (4) will hold true, hence for each $k \geq 1$, we would have the following contradictory pair of inequalities:

$$
\varepsilon_{1} \cdot \frac{1}{\Delta_{F(k)}} \leq \Delta_{U}\left(x_{1} \ldots x_{F(k)}\right) \leq \varepsilon \cdot 2^{F(k)-k} .
$$

We are now able to deduce Chaitin's information-theoretic incompleteness theorem from the uncertainty relation (4). Assume by absurdity that $Z F C$ can determine infinitely many digits of $\Omega_{U}=0 . \omega_{1} \omega_{2} \ldots$ Then, we could enumerate an infinite sequence of digits of $\Omega_{U}$, thus contradicting the above theorem.

In particular, there exists a bound $N$ such that $Z F C$ cannot determine more than $N$ scattered digits of $\Omega_{U}=0 . \omega_{1} \omega_{2} \ldots$.

\section{CONCLUSION}

We have shown that uncertainty implies algorithmic randomness which, in turn, implies incompleteness. Specifically, the complexity-theoretic characterization of the randomness of the halting probability of a universal self-delimiting Turing machine $U$, Chaitin Omega number $\Omega_{U}$, can be recast as a "formal uncertainty principle": an uncertainty relation between the accuracy of one's estimate of $\Omega_{U}$ and the complexity of the initial bit string. This relation implies Chaitin's information-theoretic version of Gödel's incompleteness.

The uncertainty relation applies to all self-delimiting Turing machines $C$. For the class of machines whose halting probabilities $\Omega_{C}$ are computable, we have shown that one can construct a quantum computer for which the uncertainty relation describes conjugate observables. Therefore, in these particular instances, the uncertainty relation is equivalent to Heisenberg's.

There is an important distinction between "quantum randomness" and our formal uncertainty principle. They are separate concepts. In the Copenhagen 
interpretation, the random collapse of the wave-function is a postulate. In the Bohmian interpretation, where there are real particles with real (though nonNewtonian) trajectories, randomness comes from our ignorance about the system; the velocity of any particle depends instantaneously on every other particle. In one case the interpretation is probabilistic, while in the other, it is completely deterministic. We cannot distinguish between these. Our result concerns a different source of randomness.

Like Heisenberg's uncertainty principle, our formal uncertainty principle is a general one; they both apply to all systems governed by the wave equation, not just quantum waves. We could, for example, use sound waves instead of a quantum system by playing two pure tones with frequencies $f$ and $f+\Delta_{C}\left(\omega_{1} \ldots \omega_{s}\right)$. Then $\Delta_{s}$ corresponds to the complementary observable, the length of time needed to perceive a beat. The (algorithmic) randomness we are concerned with seems to be pervasive in physics, even at the classical level. We may speculate that uncertainty implies randomness not only in mathematics, but also in physics.

\section{ACKOWLEDGEMENT}

We thank K. Svozil for suggesting the references (Casti and Traub, 1994; Casti and Karlquist, 1996) and speculating that "uncertainty implies randomness in physics."

\section{REFERENCES}

Barrow, J. D. (1998). Impossibility: The Limits of Science and the Science of Limits, Oxford University Press, Oxford.

Barrow, J. D. (2000). Mathematical jujitsu: Some informal thoughts about Gödel and physics.' Complexity 5(5), 28-34.

Calude, C. S. (2002a). Information and Randomness: An Algorithmic Perspective, Revised and Extended, 2nd edn. Springer Verlag, Berlin.

Calude, C. S. (2002b). Chaitin $\Omega$ numbers, Solovay machines and incompleteness. Theoretical Computer Science 284, 269-277.

Calude, C. S. (2002c). Incompleteness, complexity, randomness and beyond. Minds and Machines: Journal for Artificial Intelligence, Philosophy and Cognitive Science 12(4), 503-517.

Calude, C. S. and Pavlov, B. (2002). The Poincaré-Hardy inequality on the complement of a Cantor set. In lpay, D., Gohberg, I., and Vinnikov, V., eds., Interpolation Theory, Systems Theory and Related Topics, Operator Theory: Advances and Applications, Vol. 134, pp. 187-208. Birkhäuser Verlag, Basel.

Casti, J. L. and Traub, J. F., eds. (1994). On Limits. Santa Fe Institute Report 94-10-056, Santa Fe, NM.

Casti, J. L. and Karlquist, A., eds. (1996). Boundaries and Barriers. On the Limits to Scientific Knowledge. Addison-Wesley, Reading, MA.

Chaitin, G. J. (1975a). A theory of program size formally identical to information theory. Journal of the Association for Computing Machinery 22, 329-340. (Received April 1974) (Reprinted in: Chaitin, 1990, pp. 113-128). 
Chaitin, G. J. (1975b). Randomness and mathematical proof. Scientific American 232(5), 47-52.

Chaitin, G. J. (1982). Gödel's theorem \& information. International Journal of Theoretical Physics 22, 941-954.

Chaitin, G. J. (1990). Information, Randomness and Incompleteness, Papers on Algorithmic Information Theory, 2nd edn. World Scientific, Singapore.

Chaitin, G. J. (1992). Information-Theoretic Incompleteness. World Scientific, Singapore.

Chaitin, G. J. (1999). The Unknowable. Springer Verlag, Singapore.

Chaitin, G. J. (2002). Computers, paradoxes and the foundations of mathematics. American Scientist 90, 164-171.

Delahaye, J.-P. (1994). Information, Complexité et Hasard. Hermes, Paris.

Deutsch, D. (1997). The Fabric of Reality. Allen Lane, Penguin Press, New York.

Feferman, S., Dawson, J. Jr., Kleene, S. C., Moore, G. H., Solovay, R. M., van Heijenoort, J., eds. (1986). Kurt Gödel Collected Works, Vol. I. Oxford University Press, New York.

Feferman, S., Dawson, J. Jr., Kleene, S. C., Moore, G. H., Solovay, R. M., van Heijenoort, J., eds. (1990) Kurt Gödel Collected Works, Vol. II. Oxford University Press, New York.

Gödel, K. (1931). Über formal unentscheidbare Sätze der Principia Mathematica und verwandter. Systeme Monatshefte für Mathematik und Physik 38, 173-198 (Received 17 November 1930).

Geroch, R. and Hartle, J. B. (1986). Computability and physical theories. Foundations of Physics 16(6), 533-550.

Hawking, S. W. (2002). Gödel and the end of physics. Dirac Centennial Celebration, Cambridge, UK, July 2002, http://www.damtp.cam.ac.uk/strtst/dirac/hawking/.

Heisenberg, W. (1926). Quantenmechanik. Die Naturwissenschaften 14, 899-894.

Heisenberg, W. (1927). Über den Anschaulichen Inhalt der Quantentheoretischen Kinematik und Mechanik. Zeitschrift für Physik 43, 172-198. (Received 23 March 1927) English translation. In Wheeler, J. A., and Zurek, H. eds., Quantum Theory and Measurement, pp. 62-84. Princeton University Press, Princeton, 1983.

Kennard, E. H. (1927). Zur Quantenmechanik einfacher Bewegungstypen. Zeitschrift für Physik 44, 326-352.

Pauli, W. (1979). In Hermann, A., von Meyenn, K., and Weiskopf, V. F., eds., Wissentschaftlicher Briefwechsel mit Bohr, Einstein, Heisenberg u.a. Volume 1 (1919-1929). Springer-Verlag, Berlin.

Peres, A. (1985). Einstein, Gödel, Bohr. Foundations of Physics 15(2), 201-205.

Peres, A. and Zurek, W. H. (1982). Is quantum theory universally valid? Am. J. Phys. 50(9), 807-810.

Solovay, R. M. (2000). A version of $\Omega$ for which $Z F C$ can not predict a single bit. In Calude, C. S., and Păun, G., eds., Finite Versus Infinite. Contributions to an Eternal Dilemma, pp. 323-334. Springer-Verlag, London.

Svozil, K. (2005). Computational universes, CDMTCS Research Report 216, May 2003; Chaos, Solitons \& Fractals 25(4), 845-859.

Svozil, K. (2004). Private communication to Calude, 8 February 2004.

Tadaki, K. (2002). Upper bound by Kolmogorov complexity for the probability in computable POVM measurement, Los Alamos preprint archive, http://arXiv:quant-ph/0212071, 11 December 2002.

Tarski, A. (1994). Introduction to Logic and to the Methodology of Deductive Sciences, 4th edn. Oxford University Press, New York. 